\title{
Fluctuating baseline pain implicated in failure of clinical trials
}

MONTREAL - In the study of pain, the gold standard for assessing discomfort and suffering in human clinical trials is simply to ask participants how much pain they are feeling. Most experts agree that this metric is flawed, but they also acknowledge the lack of suitable biomarkers or other objective alternatives to replace self-reported measurements. Given these limitations, researchers are investigating better ways to make sense of people's pain ratings to improve trial design.

In an attempt to do so, Robert Palmer from Forest Laboratories in Jersey City, New Jersey and his colleagues reevaluated data from two phase 3, randomized, double-blind trials testing the drug milnacipran versus placebo. Milnacipran, a selective serotonin and norepinephrine reuptake inhibitor marketed by Forest Labs and Cypress Bioscience under the brand name Savella, was approved in the US last year for treating fibromyalgia, a chronic condition marked by extreme muscle and connective tissue pain.

They found that among the 2,000-plus participants only around $20 \%$ of those who self-reported smaller pain fluctuations before the study treatment responded to placebo, compared to around $35 \%$ of those with the largest swings in baseline pain. The difference among those who improved with milnacipran compared to placebo was also somewhat more pronounced in individuals with steady pain, suggesting that focusing on only subjects with stable baseline pain could increase the power of the clinical trial to find significant effects. "Your ability to discriminate between the placebo and the drug is stronger in the low-variability group," says Palmer, who presented the findings at the World Congress of Pain here in August.

Even though focusing on only people with steady aches and pains could save companies a lot of time and money, scientists are not quite ready to exclude participants with variable discomfort from pain studies. "Our findings are provocative, but they're still preliminary," says Richard Harris of the University of Michigan-Ann Arbor, who has also studied fluctuating pain and milnacipran on a smaller scale (Arthritis Rheum. 52, 3670-3674, 2005). Researchers now need to verify whether the effect is true in other data sets, with other metrics of pain and in other diseases that also rely on measures of self-reported pain severity, he adds. "Baseline pain variability could be a factor that increases the imprecision of clinical trial assessment," Harris says.

Robert Dworkin, who studies the methodology of pain trials at the University of
Rochester in New York, says the recent reports are part of a growing body of data supporting the need for evidence-based trial designs in the pain field. He notes that negative trials and longer trials have also been shown to lead to greater placebo responses. Considering that the vast majority of candidate painkillers have failed in late-stage clinical trials, Dworkin says that parsing people by their baseline pain levels could be one solution to improving the field's translational success.
"Maybe we're not doing the very best we can to design trials that test these analgesics in the best way possible," says Dworkin, who last month received a \$1 million contract through the US Food and Drug Administration's new Analgesic Clinical Trial Innovations, Opportunities, and Networks initiative to study the design, implementation and interpretation of pain trials. "If we can have adequately powered trials with fewer subjects that would be a huge plus."

Elie Dolgin

\section{Talkin' 'bout my (third) generation}

In 2004, officials at the US National Human Genome Research Institute (NHGRI) set an ambitious goal: to reduce the cost of sequencing an entire human genome by four orders of magnitude within a decade. At the time, shortly after the publication of the Human Genome Project, a new three-billion-base-pair genome sequence still cost more than $\$ 10$ million. So the idea was to get the cost down to under $\$ 1,000$ by 2014 .

Thanks to next-generation sequencing, which relies on massive parallel analyses of millions of short nucleic acid fragments, whole genome sequences are already available for under $\$ 20,000$ apiece. To lower the cost further, many researchers are now vying to develop the first $\$ 1,000$ genome by refining their sequencing-by-synthesis methods or pursuing a range of newer, single-molecule, 'third generation' DNA sequencing technologies.

The multitude of candidate platforms is a good thing, says Jeffery Schloss, program director for technology development at the NHGRI, which last month announced the recipients of its seventh annual Advanced Sequencing Technology Awards. "Competition has been essential to keeping quality high and driving costs down, and we hope that continues as the new technologies emerge," he says.

Here are some of this year's more inventive ideas:

\section{Pore your heart out}

The most common strategy being pursued involves reading individual nucleotides in a single strand of DNA as it travels through atomic-sized holes known as nanopores. This approach avoids the time-consuming and expensive cyclic addition of sequencing reagents and reads the genome rapidly in real time.

\section{A fluid approach}

GnuBIO, a startup launched by Harvard University physicist David Weitz, is developing a microfluidics-based sequencer where reactions take place in picoliter-sized drops. By using only the tiniest of droplets, the company hopes to drastically reduce the amount of costly reagents needed in more traditional next-generation platforms.

\section{Come to your sensors}

Rather than relying on artificial nanostructures, University of California-San Diego biotechnologist Xiaohua Huang plans to perform single-molecule sequencing by engineering onto the surface of natural DNA polymerases sensors that monitor how the enzymes change shape as specific base types are added to the DNA strand.

\section{Taking charge}

The approach from Silicon Valley startup Caerus Molecular Diagnostics works by measuring the increased molecular charge as nucleotides are added to DNA templates attached to microscopic beads. Crucially, this method does away with standard next-gen sequencing's expensive fluorescent labels.

\section{Heavy hitters}

Scientists at Redwood City, California's Halcyon Molecular, in collaboration with University of California-Berkeley chemist Dean Toste, propose to attach heavy atoms of osmium, iridium, gold and other elements to DNA and then use transmission electron microscopy to decode the labeled DNA. Elie Dolgin 